\title{
Sualtı Doğalgaz Boru Hatlarındaki Sızıntıların Eğri Uydurma Tabanlı Alınan Sinyalin Gücü Yöntemiyle Konumlandırılması
}

\section{Locating Leakages In The Underwater Natural Gas Pipelines Using Curve Fitting Based Received Signal Strength Method}

\author{
Yiğit MAHMUTOĞLU*1,a, Kadir TÜRK ${ }^{2, b}$ \\ ${ }^{1}$ Recep Tayyip Erdoğan Üniversitesi, Elektrik ve Elektronik Mühendisliği Bölümü, Rize \\ ${ }^{2}$ Karadeniz Teknik Üniversitesi, Elektrik-Elektronik Mühendisliği Bölümü, Trabzon
}

- Geliș tarihi / Received: 07.06.2019

- Düzeltilerek geliş tarihi / Received in revised form: 15.10 .2019

- Kabul tarihi / Accepted: 20.10 .2019

\section{Öz}

Doğalgaz rezervleri dünyanın belirli bölgelerinde bulunduğundan bu önemli enerji kaynağı talep merkezlerine ağırlıklı olarak sualtı boru hatları yardımıyla iletilmektedir. Sualtı doğalgaz boru hatları başta iç/dış korozyon olmak üzere doğa ve insan kaynaklı bazı nedenlerden dolayı delinip denize gaz sızdırabilir. Bu nedenle sualtı doğalgaz boru hatlarındaki (SDBH) sızıntıların tespiti ve konumlandırılması enerji güvenliğini sağlama ve çevre kirliliğinin önüne geçmede önemli bir yere sahiptir. Bu çalışmada, SDBH'lerde ortaya çıkabilecek deliklerin yerlerinin belirlenebilmesi amacıyla sinyal güçlerine eğri uydurmaya dayalı bir yöntem önerilmiştir. Önerilen yöntemin konumlandırma başarımı, zamanla değişen çok yollu bir sualtı akustik kanalının ve gerçek hayatta kullanılan bir sualtı doğalgaz boru hattının parametrelerinin kullanıldığı benzetim çalışmalarıyla analiz edilmiştir. Sayısal sonuçlar, bu zamanla değişen çok yollu sualtı akustik kanalı için sızıntıların alıcı sayısı, ortam gürültüsü ve deniz dalga yüksekliğine bağlı olarak kilometrelerce uzaktan konumlandırılabileceğini göstermiştir.

Anahtar kelimeler: Konum Tespiti, Pasif Akustik, Sızıntı, Sualtı Doğalgaz Boru Hattı.

\begin{abstract}
Since natural gas reserves are located in certain regions of the world, this important energy resource is transported to demand centers mainly by means of underwater pipelines. Underwater natural gas pipelines can be punctured and so the natural gas may leak into the sea due to especially internal/external corrosion and nature and man-made based reasons. For this reason, detecting and locating leakages in the underwater natural gas pipelines has an important role in ensuring energy security and preventing environmental pollution. In this study, a curve fitting to signal strength based method is proposed for positioning the leakages. The localization performance of the proposed method is analyzed by simulation studies using a time-varying multipath underwater acoustic channel and parameters of a real life underwater natural gas pipeline. Numerical results show that for the time-varying multipath underwater acoustic channel leakages can be located with low errors from kilometers away depending on receiver number, ambient noise and sea wave height.
\end{abstract}

Keywords: Localization, Passive Acoustic, Leakage, Underwater Natural Gas Pipeline.

\footnotetext{
${ }^{* a}$ Yiğit MAHMUTOĞLU; yigit.mahmutoglu@erdogan.edu.tr, Tel: (0464) 2237518 (dâhili: 1580), orcid.org/0000-0003-4409-2587

${ }^{b}$ orcid.org/0000-0002-4504-8417
} 


\section{Giriş}

Doğalgazın üretildiği bölgelerden talep merkezlerine doğru taşınması için genellikle boru hatları kullanılmaktadır. Büyük bir oranı denizlerden geçen bu boru hatları üç temel nedenden dolay1 delinip denize gaz sızdırabilmektedir. İlk neden olan başlangıç kusurları (tasarım/üretim/montaj) malzeme kusurları, kaynak kusurları ve büyük artık gerilmeler olarak tanımlanır. İkinci neden olan çevresel yükler (gaz akışı esnasında) dengesiz işletme basıncı, firtına-kasırga-dalga-akıntı, iç/dış korozyon ve deniz yatağındaki tektonik hareketleri kapsamaktadır. Üçüncü neden olan ek yükler (gaz akışı esnasında) ise gemi çapası ve balıkçılık ağlarını içermektedir (Mao vd., 2015). Sızıntı vakalarının ne siklıkta meydana geldiğine dair bir örnek olarak Meksika Körfezindeki sualtı boru hatlarında 1967-1990 yılları arasında yaşanan 1047 sızıntı verilebilir (United Nations, 2017). Başka örnekler olarak ise 1971-2000 yılları arasinda Avrupa'da sualtı boru hatlarında meydana gelen 542 sizint1 ve 2001-2011 y1llar1 arasında İngiltere'deki sualtı boru hatlarında meydana gelen 1978 sizınt1 verilebilir (United Nations, 2017; Li vd., 2016). Bu nedenle sualt1 doğalgaz boru hatlarının (SDBH) izlenmesi ve sızıntı durumunda mümkün olan en hızlı biçimde müdahale edilmesi enerji güvenliğini sağlama ve çevre kirliliğinin önüne geçmede önemli bir yere sahiptir.

Literatüre bakıldığında, SDBH'lerde oluşabilecek sızıntıların tespit edilmesi, yerlerinin belirlenmesi için içsel yöntemler ve dişsal yöntemler olarak gruplandırılan çeşitli yöntemler tanıtılmıştır. Fakat dışsal yöntem olarak gruplandırılan yöntemlerden olan pasif akustik yöntemi (PAY) dışında diğer yöntemler sualtı ortamından etkilenme, yüksek maliyetli sistem gerektirme, konum tespiti yapamama gibi çok sayıda dezavantaja sahiptir (Mahmutoglu ve Turk, 2019). SDBH'lerdeki sızıntı deliklerinin konumlarının belirlenmesi için (Mahmutoglu ve Turk, 2017, 2018a,b, 2019) çalışmalarında pasif akustik tabanlı olan çeşitli yöntemler sunulmuştur. Bu çalışmalarda tek yollu sualtı akustik kanalı göz önüne alınmıștır. (Mahmutoglu ve Turk, 2018b) çalışmasında da kanalın rastgele değişim etkisi ihmal edilmiş ancak deniz yüzeyi ve tabanından dolayı oluşan yansıma etkilerini içeren çok yollu bir sualtı akustik kanalı kullanılmıştır. Ancak gerçekçi bir sualtı akustik kanalı deniz akıntıları, deniz yüzeyinin hareketli yapısı ve verici/alıc1 hareketliliği nedeniyle değişken bir geometrik yapıya, saçılım ve Doppler kayma etkilerine sahiptir.

Bu çalışmada, (Qarabaqi ve Stojanovic, 2013a) çalışmasında önerilen ve literatürde sıklıkla kullanılan oldukça gerçekçi bir sualtı akustik kanal modeli kullanılarak, SDBH'lerdeki sizıntıların konumlandırılmasi problemi ele alınmıştır. Deniz yüzeyinin zamanla değişim etkisinin de modellendiği bu kanal modelinde akustik yayılımın birçok fiziksel özelliği göz önünde bulundurulmuştur. Çok yollu bayılma etkisine sahip bu zamanla değişen kanalda sızıntı konumlarının tespit edilmesi için literatürde önerilmiş olan pasif akustik tabanlı yöntemler yetersiz kalmaktadır. $\mathrm{Bu}$ durumda sizıntıların konumlandırılabilmesi için sualtı akustik kanalının çok yolluluk etkisiyle mücadele edilmesi gerekmektedir.

Konumlandırma literatüründe kanalın çok yolluluk etkisinin azaltılması için çeşitli çalışmalar yapılmıştır. $\mathrm{Bu}$ çalışmalardan bazılarında kullanılan erken-eksi-geç kilitlemeli çevrim, gecikme kilitlemeli çok yolluluk tahmincisi, çift delta tekniği, erken eksi geç eğimi, evre-uyumsuz gecikme kilitlemeli çok yolluluk tahmincisi ve dalgacık analizi gibi tekniklerde kanalı tanımak için vericiden alıcıya, alıcı tarafından bilinen bir eğitim dizisi gönderilmiştir (Aram vd., 2007; Marx vd., 2009; Bhuiyan ve Lohan, 2010; Tamazin vd., 2016). Başka çalışmalarda ise frekans çeşitlemesi yöntemi ve kepstrum yöntemleri kullanılmıştır (Ladha vd., 2007; Li-jun vd., 2012; Lazaro vd., 2013). Ancak bu yöntemler, doğaları gereği SDBH'lerde ortaya çıabilecek deliklerin yerlerinin tespit edilmesi için uygun değildir.

$\mathrm{Bu}$ çalışmada, SDBH'lerde ortaya çıkabilecek deliklerin yerlerinin belirlenebilmesi amaciyla ortalama sinyal güçlerine eğri uydurmaya dayalı yeni bir yöntem önerilmiştir. Bu yöntemde sızıntı deliklerinin konumlarının tespit edilmesi için doğrusal en küçük kareler (DEKK) ve ağırlıklandırılmış DEKK (ADEKK) algoritmaları kullanılmıştır. İncelemelerde (Qarabaqi ve Stojanovic, 2013a)'da önerilen gerçekçi sualtı akustik kanal modeli kullanılmıştır. Önerilen yöntemin ve algoritmaların başarımı çeşitli alıcı sayısı, ortam gürültüleri ve deniz yüzeyindeki dalga yükseklikleri için analizleri gerçekleştirilmiştir. 


\section{Sualtı Akustik Kanalı ve Ortam Gürültüsü}

\subsection{Sualtı Akustik Kanalı}

Sualtı ortamı coğrafyaya, derinliğe sualtı şekillerine göre önemli değişikliklere sahip olduğundan sualtı akustik kanal modelleri konusunda literatürde bir fikir birliği yoktur. Kullanılan bazı kanal modellerinin, kanalın rastgele değişimlerini göz ardı etmekte (Porter ve Bucker, 1987), yüksek rüzgar hızları için tutarsız sonuçlar vermekte (Heitsenrether ve Badiey, 2004) ve yüksek hesaplama karmaşıklığına sahip olmaktadır (Peterson ve Porter, 2013). Bunların haricinde, sualtı akustik kanalı için stokastik model geliştirme amacıyla da çok sayıda araştırma gerçekleştirilmiştir (Galvin ve Coats, 1996; Wang ve Yang, 2006; Radosevic vd., 2009; Socheleau vd., 2009; Qarabaqi ve Stojanovic, 2009,2011; Tomasi vd., 2010; Zhang vd., 2010). Bahsi geçen çalışmalarda genellikle sınırlı bölgelerde yapılan akustik ölçümler kullanılarak kanal modelleri oluşturulmuştur. Ancak, yukarıda da belirtildiği gibi sualtı ortamları farklı bölgelerde birbirlerinden çok farklı özellikler gösterebilir. Bu nedenle bu çalışmalarda ortaya konulan kanal modellerinin geçerli olduğu bölgeler de kısıtlıdır.

Literatürde önerilen diğer sualtı akustik kanal modelleriyle karşıslaştırılınca (Qarabaqi ve Stojanovic, 2013a)'da sualtı akustik kanalı daha gerçekçi modellenmiş olup ilgili çalışmanın yazarları kanalı tanımlamak için detaylı bir matematiksel inceleme yapmıştır. Kanal tanımlanırken akustik yayılımın fiziksel özellikleri olan alıc1/vericinin rastgele yer değiștirmeleri, Doppler etkisi, frekans tabanlı zayıflatma ve yüzey/taban yansımaları gibi bir çok etki göz ardı edilmiştir. (Qarabaqi ve Stojanovic, 2013a)'da sualtı kanalındaki değişimler, küçük ölçekli ve büyük ölçekli olarak sınıflandırılmıştır. Değişen sistem geometrisine bağlı olarak ortaya çıkan konum belirsizliğini tanımlamak için alınan sinyalin ortalama gücünü etkileyen büyük ölçekli değişimler kullanılır. Anlık kanal tepkesindeki hızlı değişimlerden sorumlu olan küçük ölçekli değişimler ise saçılım ve Doppler etkilerini tanımlamak için kullanılır. Kısaca, vericiden gönderilen sinyal sabit geometriye sahip bir kanalda (nominal kanal) alıcılara farklı varış açılarında ve farklı uzunluklara sahip yolları kat ederek ulaşacaktır. Büyük ölçekli değişimler, verici ile alıcı arasındaki yolun uzunluğunun rastgele değişmesine neden olmaktadır. Diğer taraftan küçük ölçekli değişimler ise verici ile alıcı arasindaki her bir yoldan ilerleyen sinyalin saçılmasına ve böylece çeşitli sayıda alt yola ayrılmasına neden olmaktadır. Literatürde sıklıkla kullanılan bu kanalın transfer fonksiyonu (1) denkleminde verilmiştir (Pedrosa vd., 2014; Kari vd., 2017; Zhao vd., 2017).

$H(f)=\bar{H}_{0}(f) \sum_{p} h_{p} \gamma_{p}(f) e^{-j 2 \pi f \tau_{p}}$

Burada, $\bar{H}_{0}(f), p, h_{p}, \gamma_{p}(f)$ ve $\tau_{p}$ sirasiyla referans yol için transfer fonksiyonu, yol sayısı, yol kazancı, saçılma katsayısı ve yol gecikmesidir. Değişkenler hakkındaki detaylı bilgiler ve eşitlikler (Qarabaqi ve Stojanovic, 2013a)'da verilmiştir.

Qarabaqi ve Stojanovic tarafindan önerilen bu analitik kanal modeli, yine yazarlar tarafindan deneysel çalışmalar yapılarak doğrulanmış ve (Qarabaqi ve Stojanovic, 2013b)'de tanitılan bir kanal simülatörü geliştirilmiştir. Bahsi geçen sualtı akustik kanal simülatöründe, küçük ölçekli değişimlerin benzetimleri için istatistiki denk model veya direk model kullanılmıştır. Büyük ölçekli değişimlerin etkisini ortaya koyabilmek için ise deniz tabanının ve yüzeyinin düzgün ve ses hızının sabit olduğu kabul edilerek Bellhop ya da basitleştirilmiş ışın izleme ışın seçenekleri sunulmuştur.

\subsection{Sualtı Ortam Gürülttüsü}

Sualtı ortam gürültüsü kaynakları Gauss dağılımlı olarak modellenmektedir. Türbülans $\left(\mathrm{N}_{\mathrm{t}}\right)$, gemi $\left(\mathrm{N}_{\mathrm{s}}\right)$, dalga $\left(\mathrm{N}_{\mathrm{w}}\right)$ ve termal $\left(\mathrm{N}_{\mathrm{th}}\right)$ gürültüleri sualtı ortam gürültüsünü temsil etmek için kullanılan dört ana kaynaktır. (2)-(5) denklemlerinde bu gürültülerin güç spektral yoğunluklarının ampirik formülleri $\mathrm{dB}$ re $1 \mu \mathrm{Pa} / \mathrm{Hz}$ cinsinden ve frekansin $(\mathrm{kHz}$ cinsinden) bir işlevi olarak sunulmuştur (Stefanov ve Stojanovic, 2011).

$$
\begin{aligned}
& N_{t_{-} d B}=10 \log _{10} N_{t}(f)=17-30 \log _{10}\left(f_{0}\right) \\
& N_{S_{-} d B}=10 \log _{10} N_{s}(f)=40+20(s-0.5)+26 \log _{10}\left(f_{0}\right)-60 \log _{10}\left(f_{0}+0.03\right) \\
& N_{w_{-} d B}=10 \log _{10} N_{w}(f)=50+7.5 \sqrt{w}+20 \log _{10}\left(f_{0}\right)-40 \log _{10}\left(f_{0}+0.4\right) \\
& N_{t h_{-} d B}=10 \log _{10} N_{t h}(f)=-15+20 \log _{10}\left(f_{0}\right)
\end{aligned}
$$


Burada $w \mathrm{~m} / \mathrm{sn}$ cinsinden rüzgar hızı ve $s$ gemi aktivite faktörüdür $(0 \leq s \leq 1)$. Ortam gürültüsünün toplam güç spektral yoğunluğu (6) denkleminde verilmiştir.

$$
N(f)=N_{t}(f)+N_{s}(f)+N_{w}(f)+N_{t h}(f)
$$

\section{Sızıntı Konumlandırma Yöntemi}

Oluşturulan konumlandırma sistemi Şekil 1'de gösterilmiştir. Burada hidrofonların (alıcıların) aralarında eşit mesafe bulunan şamandıralardan SDBH'nin üstüne sarkıtıldığı varsayılmıştır. Bu hidrofonlar aracılığıyla alınan akustik sinyallerin kara ortamındaki bir merkeze gönderildiği ve burada önerilen yöntem kullanılarak sızıntının konumlandırıldı̆̆ 1 varsayılmıştır. SDBH'nin izlediği yol ve derinliği bilindiğinden üç boyutlu konumlandirma problemi tek boyuta indirgenmiştir.

Hidrofonlar tarafindan alınan akustik sinyaller, sızıntı deliğinin çapı ve sualtı akustik kanalına göre değişiklik gösteren çok dar bir banda sahip işaretlerdir. Bu sebeple, (Mahmutoglu ve Turk, 2018a) çalışmasına benzer olarak, alıcı kısmında sızıntılardan dolayı oluşan akustik sinyalin bulunabileceği tüm spektrum $3 \mathrm{~Hz}$ 'lik bir bant genişliğine sahip bant geçiren filtre yardımıyla taranır.

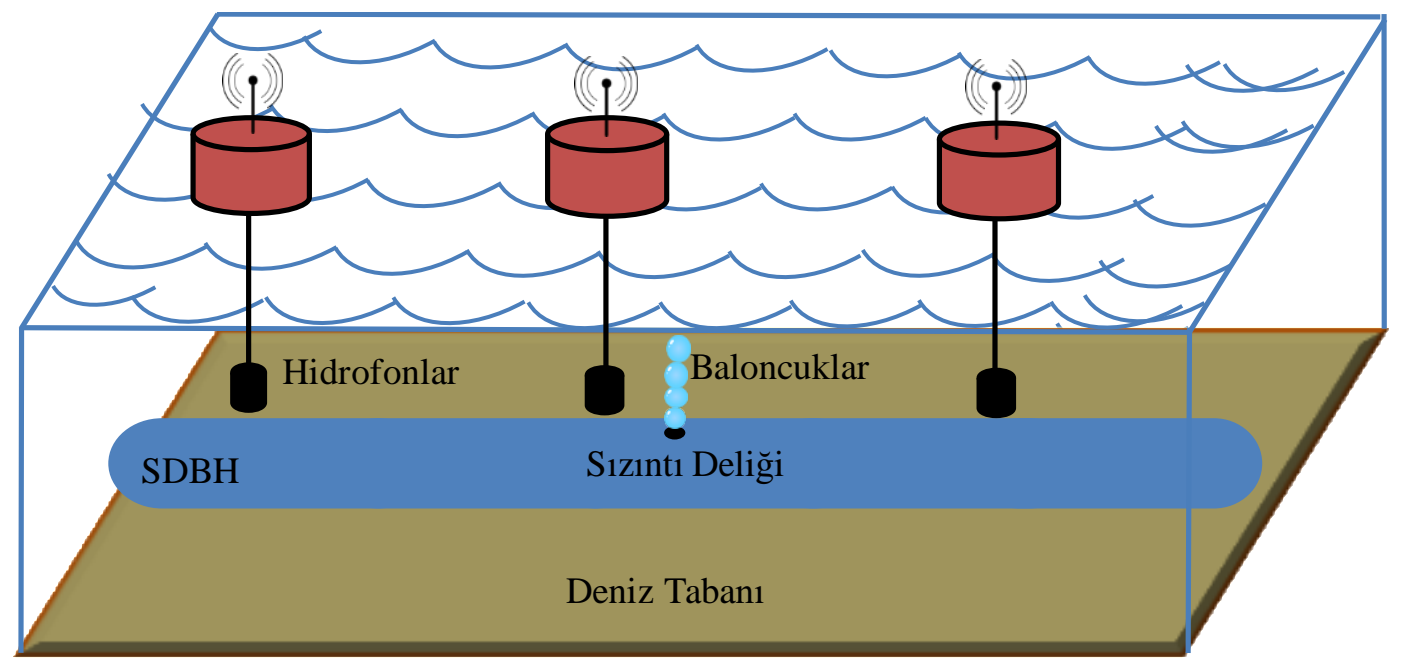

Şekil 1. Konumlandırma sistemi

Daha sonra, alınan sinyalin frekansı, benzetim sonuçları bölümünde verilen parametreler, (Mahmutoglu ve Turk, 2018a)'da verilen eşitlikler ve (7) denklemi kullanılarak delikten çıkan akustik sinyalin gücü hesaplanabilir. Filtreden geçirilen sinyallerin güçleri kaydedilir ve belirli bir süre boyunca ortalamaları alınır. Böylece ortam gürültüsünün sinyal üzerindeki etkisi azaltılmış olur.

$P=\sum_{i=1}^{B} P_{0} e^{-\left(\pi \delta f_{0}\left(t-\tau_{i}\right)\right)} \cos \left(2 \pi f_{0}\left(t-\tau_{i}\right)-\vartheta\right)$

Burada, $P, \delta, \vartheta, P_{0}, t, B, f_{0}$ ve $\tau_{i}$ sürekli bir baloncuk dizisi için akustik basınç sinyali, sönüm katsayısı, faz açısı, akustik basınç sinyalinin tepe değeri, zaman, salınım yapan baloncuk sayısı, salınım frekansı, 1. ve i. baloncuklar arasındaki zaman gecikmesidir. Bu değişkenler hakkındaki detaylı bilgiler ve eşitlikler (Mahmutoglu ve Turk, 2018a)'da verilmiştir.
Her ne kadar delikten çıkan ve alınan akustik sinyallerin güçleri bilinse de zamanla değişen çok yollu sualtı akustik kanalının neden olduğu bayılma etkisinden dolayı sızıntıyı düşük hatalı olarak konumlandırmak mümkün değildir. $\mathrm{Bu}$ nedenle sızıntının konumunu tespit etmek için sinyal gücü tabanlı eğri uydurma yöntemi önerilmiştir. Bahsi geçen yöntemde, alıcıya ulaşan sinyal yukarıda verilen filtreleme ve ortalama alarak gürültü azaltma safhalarından geçirilir.

Blok diyagramı Şekil 2'de verilmiş olan yöntem aşağıdaki gibi maddeler halinde açıklanabilir.

1- Sızıntıyı oluşturan delik ile alıcı arasındaki mesafe kısa aralıklarla değiştirilerek tüm boru hattı taranır.

2- Sualt1 akustik kanal modeline göre akustik sinyalin delikten çıkış gücü hesaplanabildiğinden, her mesafe için alıcılara ulaşması beklenen zamanla değişen gürültüsüz sinyalin gücü kaydedilir ve 
ortalaması hesaplanır. Bekleneceği sızıntı deliği ve alıcı mesafesi büyüdükçe alınan sinyalin gücü kanal sebebiyle ortaya çıkan bayılma nedeniyle düzensiz bir azalma gösterir. $\mathrm{Bu}$ düzensiz azalma ise yüksek konumlandırma hatalarına neden olur.

3- Bayılma ile oluşan düzensiz azalma etkisini azaltmak için kaydedilen delik-alıcı arası mesafeye göre değişen ortalama sinyal gücü veri setine (eğrisi), Gauss modeline göre eğri uydurulur.
4- Sızıntı deliği için konumlandırma yapılırken alıcılar tarafından alınan sinyallerin ortalama güçlerine karşılık gelen Gauss modeline göre elde edilmiş eğrideki mesafe değerleri delik ile alıcılar arasındaki uzaklığı vermektedir.

5- DEKK veya ADEKK algoritmaları kullanılarak sızıntı deliğinin konumu tespit edilir.

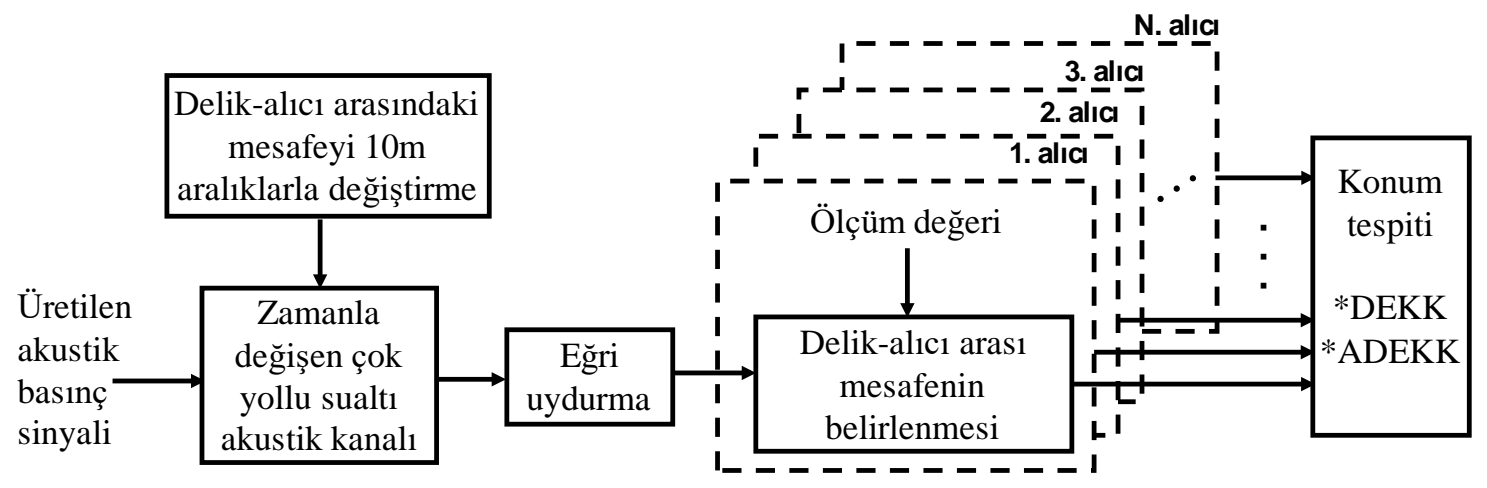

Şekil 2. Konumlandırma yönteminin blok diyagramı

Tek boyutlu uzayda konumu bilinmeyen deliğin yeri $x$ ve konumları bilinen alıcıların yerleri $\mathrm{x}_{\mathrm{z}}(\mathrm{z}=1,2, \cdots, \mathrm{m})$ ile verildiğinde sızıntı deliği ile alıcı arasındaki uzaklık $\mathrm{d}_{\mathrm{z}}=\sqrt{\left(\mathrm{x}_{\mathrm{z}}-\mathrm{x}\right)^{2}}$ şeklinde elde edilebilir. Elde edilen eğri kullanılarak bulunan sızıntı deliği ile alıcı arasındaki uzaklık $\widehat{\mathrm{d}}_{\mathrm{z}}$ olmak üzere, konumu bilinmeyen deliğin yeri DEKK algoritmasına göre (8) denklemi kullanılarak bulunabilir (Yang ve Chen, 2009; Wang, 2015).

$\hat{x}=\left(A^{T} A\right)^{-1} A^{T} b$

Yukarıdaki denklemde $A$ ve $b$ değişkenleri sırasıyla (9) ve (10) eşitliklerinde sunulmuştur.

$A=\left[\begin{array}{c}x_{1}-\frac{1}{m} \sum_{z=1}^{m} x_{z} \\ \vdots \\ x_{1}-\frac{1}{m} \sum_{z=1}^{m} x_{z}\end{array}\right]$

$b=0.5\left[\begin{array}{c}\left(x_{1}^{2}-\frac{1}{m} \sum_{z=1}^{m} x_{z}^{2}\right)-\left(\hat{d}_{1}^{2}-\frac{1}{m} \sum_{z=1}^{m} \hat{d}_{z}^{2}\right) \\ \vdots \\ \left(x_{m}^{2}-\frac{1}{m} \sum_{z=1}^{m} x_{z}^{2}\right)-\left(\hat{d}_{m}^{2}-\frac{1}{m} \sum_{z=1}^{m} \hat{d}_{z}^{2}\right)\end{array}\right]$

ADEKK algoritmasıyla bilinmeyen delik konumu, (9), (10) ve (11) denklemlerinde verilen matrisler (12) denkleminde yerine yazılarak bulunur (Zekavat ve Buehrer, 2011; Wang, 2015). $\left[4\left(\frac{1}{m} \sum_{z=1}^{m} \hat{d}_{z}\right)^{2} \sigma^{2}+2 \sigma^{4}+\operatorname{diag}\left(4 \sigma^{2} \hat{d}_{1}^{2}+\right.\right.$

$\left.\left.2 \sigma^{4}, \cdots, 4 \sigma^{2} \hat{d}_{m}^{2}+2 \sigma^{4}\right)\right]^{-1}$

Burada $W$ ağırlıklandırma matrisi ve $\sigma^{2}$ alıcılardaki gürültünün varyansıdır. $\operatorname{diag(.)}$ köşegen matrisi ifade etmektedir.

$\hat{x}=\left(A^{T} W A\right)^{-1} A^{T} W b$

\section{Benzetim Sonuçları}

Önerilen yöntemin konum tespiti performansını incelemek için oluşturulan benzetim platformunda gerçek bir SDBH'nin 10 km'lik kısmı ele alınmıştır. $400 \mathrm{~m}$ derinlikten geçen bu SDBH 136 $\mathrm{m}^{3} / \mathrm{sn}$ 'lik gaz akış hızına sahiptir (Kandiyoti, 2009). Tablo 1'de verilen parametreler, (Mahmutoglu ve Turk, 2018a)'da verilen eşitlikler ve (7) denklemi kullanılarak birçok baloncuğun sürekli olarak oluştuğu durum için akustik sinyal elde edilmiştir.

Bu çalışmada, (Qarabaqi ve Stojanovic, 2013b)‘de önerilen kanal simülatörü sualtı akustik kanalını oluşturmak için kullanılmıştır. Yukarıda da belirtildiği gibi bu simülatörde büyük ölçekli değişimlerin etkisini ortaya koyabilmek için ise deniz tabanının ve yüzeyinin düzgün ve ses 
hızının sabit olduğu kabul edilerek Bellhop yada basitleştirilmiş 1şın izleme 1şın seçenekleri sunulmuştur. Deniz taban yapısı ve ses hızı profili (ses hızının derinlikle değişimi) bölgeden bölgeye farkl1l1k göstereceğinden, belirli bir deniz taban yapısı ve ses hızı profili için ışın izleme işlemini gerçekleştiren Bellhop yerine daha genel olması sebebiyle basitleştirilmiş 1şı in izleme kullanılmıştır. Küçük ölçekli etkileri ortaya koymak için direk model kullanılmıştır.

Tablo 1. Varsayılan parametreler

\begin{tabular}{|l|l|}
\hline Parametre & Değer \\
\hline$g$ (yer çekimi ivmesi) & $9.8 \mathrm{~m} / \mathrm{sn}^{2}$ \\
\hline$h$ (derinlik) & $400 \mathrm{~m}$ \\
\hline$\rho$ (deniz suyunun yoğunluğu) & $1000 \mathrm{~kg} / \mathrm{m}^{3}$ \\
\hline$\kappa$ (özel ısı oranı) & 1.49 \\
\hline $\begin{array}{l}\tau \text { (baloncuklar arasındaki zaman } \\
\text { gecikmesi) }\end{array}$ & $0.01975 \mathrm{sn}$ \\
\hline$d_{\text {ref }}$ (referans mesafe) & $1 \mathrm{~m}$ \\
\hline$d_{\text {del }}$ (sızıntı deliğini çapı) & $1 \mathrm{~mm}$ \\
\hline$S$ (yüzey gerilmesi) & $0.068 \mathrm{~N} / \mathrm{m}$ \\
\hline$Q$ (gaz akış hızı) & $136 \mathrm{~m}^{3} / \mathrm{sn}$ \\
\hline
\end{tabular}

Simülatörde kullanılan kanal, yüzey dalga, büyük ve küçük ölçekli etkiler için kullanılan benzetim parametreleri değerleri Tablo 2 ve Tablo 3 'te verilmiştir. İlgili denklemler (Qarabaqi ve Stojanovic, 2013a)'da yer almaktadır.

Tablo 2. Yüzey dalga ve kanal değişkenleri değerleri.

\begin{tabular}{|l|l|}
\hline Değişken & Değeri \\
\hline Alıcı derinliği & $398 \mathrm{~m}$ \\
\hline Boru hattı ve deniz derinliği & $400 \mathrm{~m}$ \\
\hline Delik - alıcı arası mesafe & $0-10 \mathrm{~km}$ \\
\hline Sızıntı deliğinin derinliği & $400 \mathrm{~m}$ \\
\hline Sudaki ses hızı & $1500 \mathrm{~m} / \mathrm{sn}$ \\
\hline Yayılım faktörü & 2 \\
\hline Deniz tabanının yoğunluğu & $1800 \mathrm{~kg} / \mathrm{m}^{3}$ \\
\hline Deniz tabanındaki ses hızı & $1200 \mathrm{~m} / \mathrm{sn}$ \\
\hline Kesim faktörü & 20 \\
\hline Suyun yoğunluğu & $1000 \mathrm{~kg} / \mathrm{m}^{3}$ \\
\hline Minimum frekans & $49.7 \mathrm{~Hz}$ \\
\hline Bant genişliği & $3 \mathrm{~Hz}$ \\
\hline Frekans çözünürlüğü & $0.003 \mathrm{~Hz}$ \\
\hline Zaman çözünürlüğü & $0.1 \mathrm{~s}$ \\
\hline Dalga genliği (yüksekliği, $A w)$ & $1 \mathrm{~m}, 5 \mathrm{~m}$ \\
\hline Dalga frekansı & $0.1 \mathrm{~Hz}$ \\
\hline
\end{tabular}

Dalga yüksekliğinin $1 \mathrm{~m}$ ve $5 \mathrm{~m}$ olduğu durumlarda sızıntının oluştuğu delik ile alıcı arasındaki yolda $10 \mathrm{~m}$ aralıklarla boru hatt1 boyunca noktalar belirlenmiş ve bu noktalardaki zamanla değişen gürültüsüz alınan sinyallerin güçlerinin ortalamaları hesaplanmıştır. Elde edilen ortalama sinyal gücü veri dizileri üç terimli olarak Gauss modeline uydurulmuştur. Şekil 3'te dalga yüksekliğinin $1 \mathrm{~m}$ ve $5 \mathrm{~m}$ olduğu durumlar için elde edilmiş (ana) veri dizileri ve bu dizilere uydurulan eğriler sunulmuştur. Benzetimler boyunca, ortam gürültüsünün alıcılarda oluşturduğu gürültü gücü $3 \mathrm{~Hz}$ bant genişliği esas alınarak hesap edilmiştir. Sızıntının oluştuğu deliğin yeri $0-10 \mathrm{~km}$ arasında $10 \mathrm{~m}$ aralıklarla değiştirilmiştir.

Tablo 3. Büyük ölçekli ve küçük ölçekli etkiler için kullanılan değişken değerleri.

\begin{tabular}{|c|c|}
\hline Değişken & Değeri \\
\hline Alıcı yüksekliğinin değişim aralığı & {$\left[\begin{array}{ll}0 & 5\end{array}\right] \mathrm{m}$} \\
\hline $\begin{array}{l}\text { Deniz yüzeyindeki büyük ölçek yüksek } \\
\text { değişimlerinin standart sapması }\end{array}$ & 1 \\
\hline Deniz yüzey yüksekliğinin değişim aralığı & {$\left[\begin{array}{lll}-10 & 10\end{array}\right] \mathrm{m}$} \\
\hline Toplam benzetim sinyali süresi & $80 \mathrm{sn}$ \\
\hline Kanal mesafesinin değişim aralığ & {$\left[\begin{array}{lll}-20 & 20\end{array} \mathrm{~m}\right.$} \\
\hline Büyük ölçekli değişimler için AR & 0.9 \\
\hline $\begin{array}{l}\text { Deniz yüzeyindeki küçük ölçekli yüzey } \\
\text { değişimlerinin varyans1 }\end{array}$ & 1.125 \\
\hline $\begin{array}{l}\text { Kanal boyunun büyük ölçekli değişimleri } \\
\text { için standart sapması }\end{array}$ & 1 \\
\hline $\begin{array}{l}\text { Küçük ölçekli değişimlerde bağdaşım } \\
\text { süresi }\end{array}$ & $40 \mathrm{sn}$ \\
\hline $\begin{array}{l}\text { Alt yollardaki gecikmelerin 3-dB güç } \\
\text { spektral yoğunlukları genişliği }\end{array}$ & 0.0005 \\
\hline $\begin{array}{l}\text { Küçük ölçekli taban değişimlerinin } \\
\text { varyansı }\end{array}$ & 0.5625 \\
\hline Alt yol genliklerinin ortalaması & 0.025 \\
\hline $\begin{array}{l}\text { Alıcı yüksekliğinin büyük ölçek } \\
\text { değişimlerinin standart sapması }\end{array}$ & 1 \\
\hline Alt yol genliklerinin varyans1 & 0.000006 \\
\hline Alt yol sayısı & 20 \\
\hline
\end{tabular}
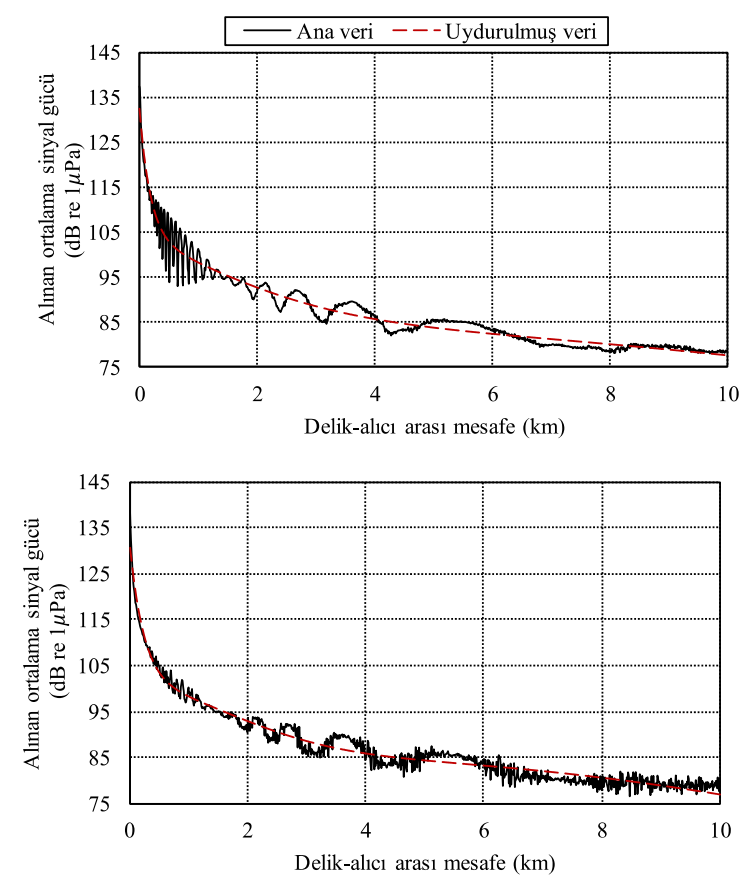

Şekil 3. Dalga yükseklikleri $1 \mathrm{~m}$ (üstte) ve $5 \mathrm{~m}$ (altta) için hesaplanan veri setleri ve bunlara üç terimli olarak Gauss modeline göre uydurulan eğriler. 
Önerilen yöntemin konumlandırma başarımı farklı dalga yüksekliği, ortam gürültüsü ve alıcı sayısı olmak üzere üç değişik durum için incelenmiştir. Farklı alıc1 sayısı durumu haricindeki diğer durumlarda alıcı sayısı 4 ve bu alıcıların yerleri ( 2 $\mathrm{km}, 4 \mathrm{~km}, 6 \mathrm{~km}, 8 \mathrm{~km}$ ) olarak seçilmiştir. Bu çalışmada ele alınan beş farklı ortam gürültüleri olan $N_{1}, N_{2}, N_{3}, N_{4}$ ve $N_{5}$ daha önce bahsedilen tüm ortam gürültüsü bileşenlerini içermektedir. Bahsedilen ortam gürültüleri Tablo 4'te verilmiştir. Burada $w \mathrm{~m} / \mathrm{s}$ cinsinden rüzgâr hızı ve GAF gemi aktivite faktörüdür.

Tablo 4. Ortam gürültüleri

\begin{tabular}{|c|c|c|}
\hline $\begin{array}{c}\text { Ortam } \\
\text { gürültüsü }\end{array}$ & $\begin{array}{c}\text { w }(\mathrm{m} / \mathrm{s}) \text { ve } \\
\mathrm{GAF}\end{array}$ & Güç $(\mathrm{dB}$ re $1 \mu \mathrm{Pa})$ \\
\hline$N_{1}$ & 19 ve 0.1 & 80.75 \\
\hline$N_{2}$ & 19 ve 0.3 & 83.87 \\
\hline$N_{3}$ & 19 ve 0.5 & 87.46 \\
\hline$N_{4}$ & 19 ve 0.7 & 91.3 \\
\hline$N_{5}$ & 19 ve 1 & 97.2 \\
\hline
\end{tabular}

Şekil 4'te dalga yüksekliğinin $1 \mathrm{~m}$, ortam gürültüsünün $N_{5}$, alıcı sayısının 4 olduğu durum için DEKK ve ADEKK algoritmalarında sızıntı deliğinin konumu ile ortalama karesel konum hatasının karekökünün (OKKHK) değişimi verilmiştir. Kanalın bayılma etkisi kendisini bu eğrilerde OKKHK değerlerindeki ani düşüş ve artışlarla göstermiştir.
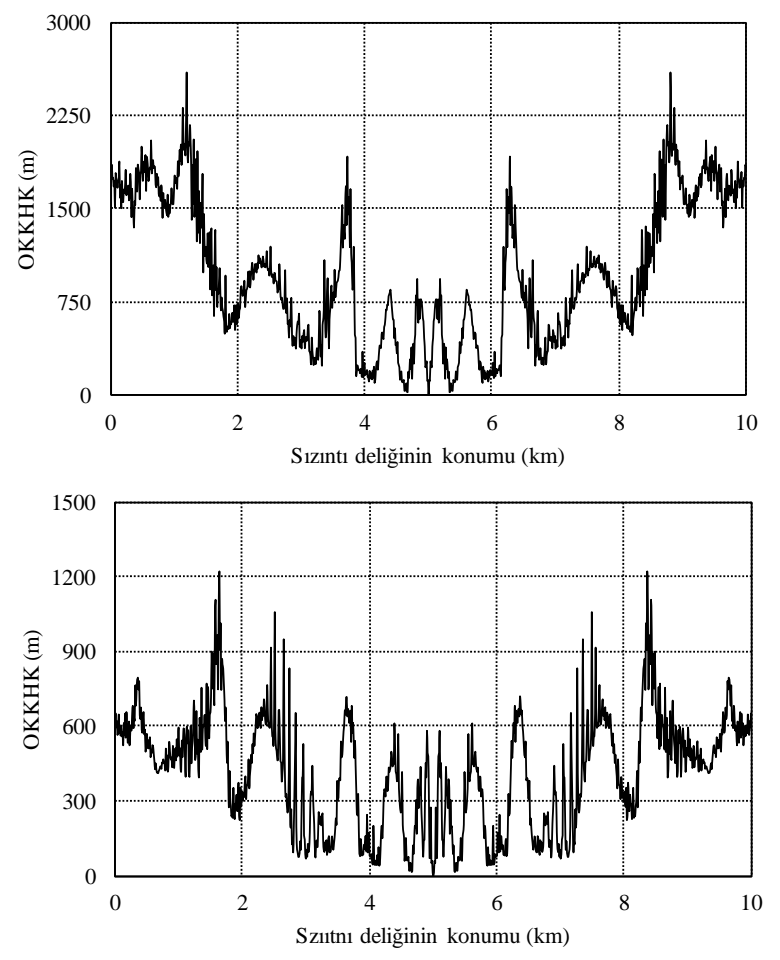

Şekil 4. DEKK (üstte), ADEKK (altta) algoritmaları, dalga yüksekliği $1 \mathrm{~m}$, alıcı sayısı 4 ve ortam gürültüsü $N_{5}$ olduğu durumda sizıntı deliğinin konumuna göre OKKHK'nin değişimi.
Farklı dalga yüksekliği durumlarında delik konumunun $10 \mathrm{~m}$ aralıkla değiştirildiği $10 \mathrm{~km}$ uzunluğundaki boruda hesaplanan OKKHK değerlerinin ortalamaları ve gemi aktivite faktörleri arasındaki ilişkiler her iki algoritma için de Şekil 5'te gösterilmiştir. $\mathrm{Bu}$ şekillerden görüldüğü üzere ADEKK'nin başarımı DEKK'den daha yüksektir. Konumlandırma doğruluğunun gemi aktivitesinin (ortam gürültü gücü) artışıyla ters orantılı olduğu görülmektedir. Buna ek olarak, dalga yüksekliğinin konum tespitini önemli ölçüde bozduğu da görülmektedir.
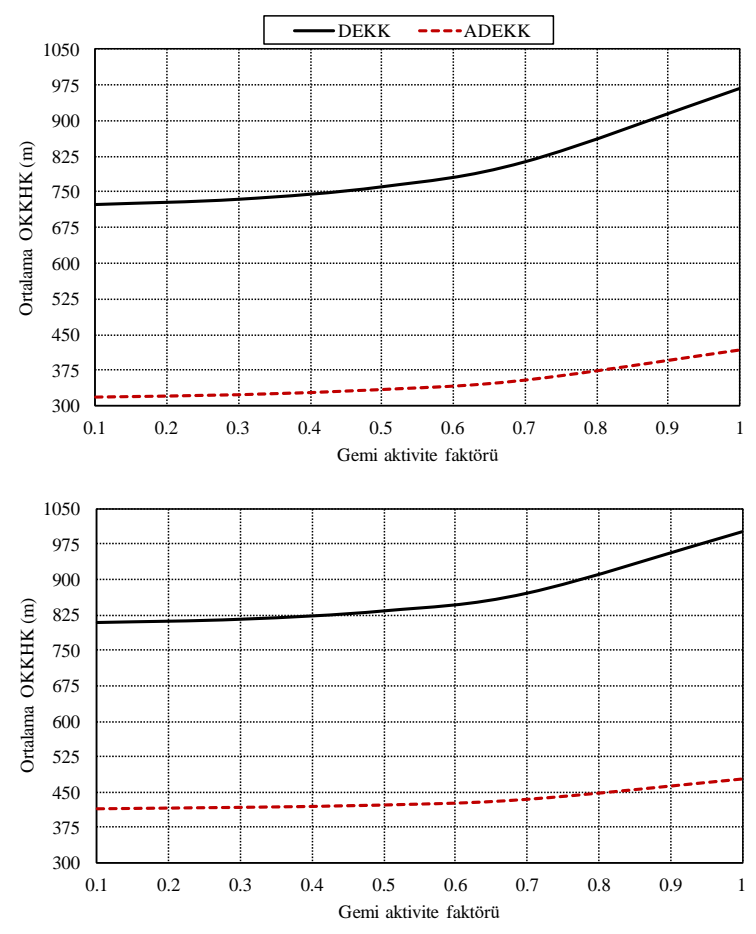

Şekil 5. Tüm algoritmalar için dalga yüksekliklerinin $1 \mathrm{~m}$ (üstte) ve $5 \mathrm{~m}$ (altta) olduğu durumda ortalama OKKHK'nın gemi aktivite faktörüne göre değişimi.

Ortam gürültüleri $N_{1}, N_{3}, N_{5}$, dalga yüksekliği 5 $\mathrm{m}$ için DEKK, ADEKK algoritmalarının konum hatalar1 ve bu konum hatalarının olasılık yoğunlukları arasındaki ilişkiler Şekil 6'da sunulmuştur. Tablo 5'te ise dalga yüksekliklerinin $1 \mathrm{~m}$ ve $5 \mathrm{~m}$, ortam gürültülerinin $N_{1}, N_{3}, N_{5}$ olduğu durumda DEKK, ADEKK algoritmaları ile elde edilen 500 m'den düşük konumlandırma hatalarının olma ihtimalleri sunulmuştur.

Alıcı sayısının 3, 4, 5 ve 6 olduğu durumlar incelenmiştir. $\mathrm{Bu}$ alıcılar10 km'lik hat üzerinde sirasiyla $(3 \mathrm{~km}, 5 \mathrm{~km}, 7 \mathrm{~km}),(2 \mathrm{~km}, 4 \mathrm{~km}, 6 \mathrm{~km}, 8$ $\mathrm{km}),(1 \mathrm{~km}, 3 \mathrm{~km}, 5 \mathrm{~km}, 7 \mathrm{~km}, 9 \mathrm{~km})$ ve $(1 \mathrm{~km}$, $2.6 \mathrm{~km}, 4.2 \mathrm{~km}, 5.8 \mathrm{~km}, 7.4 \mathrm{~km}, 9.8 \mathrm{~km})$ konumlarına yerleştirilmiştir. 
Tablo 5. $1 \mathrm{~m}$ ve $5 \mathrm{~m}$ dalga yükseklikleri, $N_{1}, N_{3}$, $N_{5}$ ortam gürültüleri ve DEKK, ADEKK algoritmaları için konum hatalarının 500 m'den düşük olma olasılıkları.

\begin{tabular}{|l|cll|}
\hline \multirow{4}{*}{$A w=1 \mathrm{~m}$} & Gürültü & DEKK & ADEKK \\
\cline { 2 - 4 } & $N_{1}$ & $\% 40.4$ & $\% 78.62$ \\
& $N_{3}$ & $\% 37.7$ & $\% 77.26$ \\
& $N_{5}$ & $\% 36$ & $\% 71.7$ \\
\hline \multirow{4}{*}{$A w=5 \mathrm{~m}$} & Gürültü & DEKK & ADEKK \\
\cline { 2 - 4 } & $N_{1}$ & $\% 36.48$ & $\% 69.64$ \\
& $N_{3}$ & $\% 34.76$ & $\% 69.53$ \\
& $N_{5}$ & $\% 33.64$ & $\% 67.69$ \\
\hline
\end{tabular}
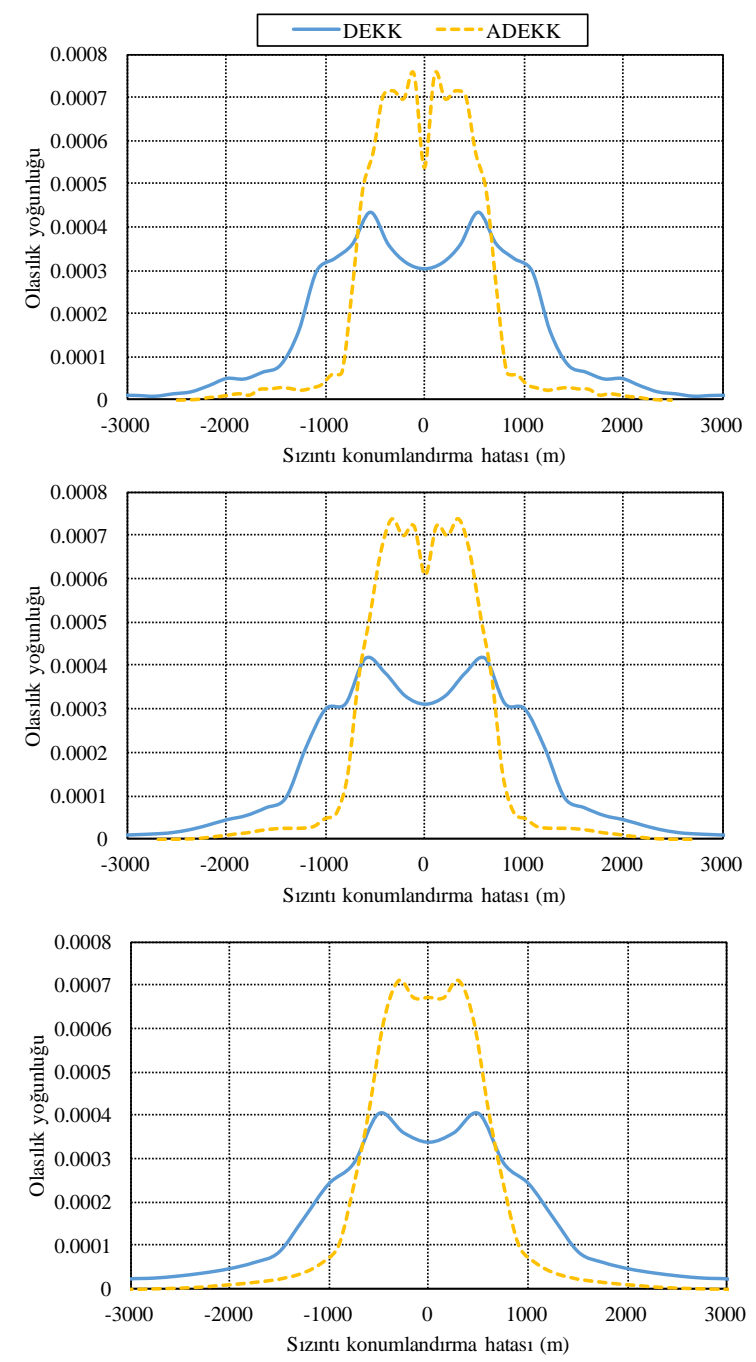

Şekil 6. $5 \mathrm{~m}$ dalga yüksekliği durumunda $N_{1}$ (üstte), $N_{3}$ (ortada), $N_{5}$ (altta) ortam gürültüleri ve her iki algoritma için konum hatası ve olasılık yoğunluğu arasındaki ilişkiler.

Çeşitli alıcı sayıları için sızıntı delik konumunun $10 \mathrm{~m}$ aralıklarla değiştirilerek elde edilen OKKHK'lerin ortalamalarının değişimi, dalga yüksekliğinin $5 \mathrm{~m}$, ortam gürültüsünün $N_{5}$ olduğu durumda DEKK, ADEKK algoritmaları için Şekil 7'de sunulmuştur.

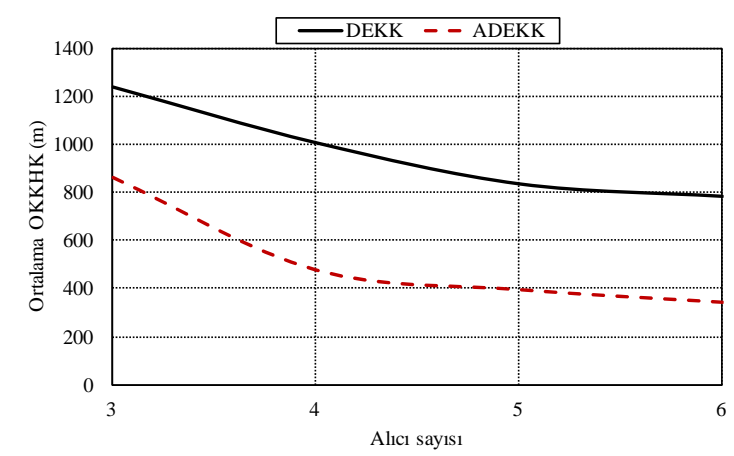

Şekil 7. Dalga yüksekliği $5 \mathrm{~m}$, ortam gürültüsü $N_{5}$ için ortalama OKKHK'nın alıcı sayısına göre değişimi.

\section{Sonuçlar}

$\mathrm{Bu}$ çalışmada, sualtı doğalgaz boru hatlarında ortaya çıabilecek deliklerin yerlerinin belirlenebilmesi amaciyla ortalama sinyal güçlerine eğri uydurmaya dayalı yeni bir yöntem önerilmiştir. Önerilen yöntemle DEKK ve ADEKK algoritmaları kullanılarak sızıntı deliği konumlandırılmıştır. Yöntemin konumlandırma başarımını ortaya koymak için yapılan benzetim çalışmalarında gerçek hayatta kullanılan bir SDBH göz önüne alınmış ve oldukça gerçekçi olan çok yollu zamanla değişen bir sualtı akustik kanal modeli kullanılmıştır. Yapılan benzetim çalışmaları sonucunda, $10 \mathrm{~km}$ uzunluğa sahip bu SDBH'deki sızıntının farklı dalga yüksekliği ve ortam gürültüsü durumlarında DEKK ve ADEKK algoritmaları için sırasıyla (4 alıcı için) \%33.64 ile $\% 40.4$ ve \%67.69 ile \%78.62 arasında değişen olasılıklarla 500 m'den düşük hatalarla konumlandırılabileceği gösterilmiştir.

\section{Kaynaklar}

Aram, M., El-Rabbany, A., Krishnan, S. ve Anpalagan, A., 2007. Single Frequency Multipath Mitigation Based On Wavelet Analysis. Journal of Navigation 60, 281-290.

Bhuiyan, M.Z.H. ve Lohan, E.S., 2010. Advanced Multipath Mitigation Techniques for SatelliteBased Positioning Applications. International Journal of Navigation and Observation, 2010, 115.

Galvin, R. ve Coats, R.E.W., 1996. A stochastic underwater acoustic channel model, Proceedings of MTS/IEEE OCEANS Conference, September 1996, Florida, USA, s. 203-210.

Heitsenrether, R. M. ve Badiey, M., 2004. Modeling acoustic signal fluctuations induced by sea surface roughness, Proceedings of High 
Frequency Ocean Acoustics Conference, March 2004, California, USA, s. 214-221.

Kandiyoti, R., 2009. Under the sea. Engineering \& Technology, 4, 26-28.

Kari, D., Marivani, I., Khan, F., Sayin, M. O. ve Kozat, S. S., 2017. Robust adaptive algorithms for underwater acoustic channel estimation and their performance analysis. Digital Signal Processing, 68, 57-68.

Ladha, C., Sharif, B. S. ve Tsimenidis, C. C., 2007. Mitigating propagation errors for indoor positioning in wireless sensor networks, IEEE International Conference on Mobile Adhoc and Sensor Systems (MASS), October 2007, Pisa, Italy, s. 8-11.

Lazaro, A., Girbau, D., Moravek, P. ve Villarino, R., 2013. A Study on Localization in Wireless Sensor Networks using Frequency Diversity for Mitigating Multipath Effects. ELEKTRONIKA IR ELEKTROTECHNIKA, 19, 82-87.

Li, X., Chen, G. ve Zhu, H., 2016. Quantitative risk analysis on leakage failure of submarine oil and gas pipelines using Bayesian networg. Process Safety and Environmental Protection, 103, 163173.

Li-Jun, C., Xiang, G. ve Liang, A., 2012. Multipath passive localization in shallow water channel. Journal of Nanjing University (Natural Sciences), 48, 609-615.

Mahmutoglu, Y. ve Turk, K., 2017. Remote leak hole localization for underwater natural gas pipelines, 40th Telecommunications and Signal Processing Conference, July 2017, Barcelona, Spain, s. 528-531.

Mahmutoglu, Y. ve Turk, K., 2018a. A passive acoustic based system to locate leak hole in underwater natural gas pipelines. Digital Signal Processing, 76, 59-65.

Mahmutoglu, Y. ve Turk, K., 2018b. Localization of Leakages in Underwater Natural Gas Pipelines for Multipath Propagation, 26th Signal Processing and Communications Application Conference, May 2018, İzmir, Turkey, s. 1-4.

Mahmutoglu, Y. ve Turk, K., 2019. Received signal strength difference based leakage localization for the underwater natural gas pipelines. Applied Acoustics, 153, 14-19.

Mao, D., Chu, G., Yanga, L. ve Li, Z., 2015, Deepwater Pipeline Damage and Research on Countermeasure. Aquatic Procedia, 3, 180-190.

Marx, M., Kokozinski, R. ve Müller, H.C., 2009. Time Synchronization for Real Time Localization
Systems with Multi Path Mitigation, IEEE MTT-S International Microwave Workshop on Wireless Sensing, Local Positioning and RFID,September 2009, Cavtat, Croatia, s. 1-4.

Pedrosa, P., Dinis, R. ve Nunes, F. 2014., Joint Equalization and Phase Drift Estimation for Underwater Acoustic Communications, IEEE Global Communications Conference, December 2014, Texsas, USA, s. 4096-4101.

Peterson, J.C. ve Porter, M.B., 2013. Ray/beam tracing for modeling the effects of ocean and platform dynamics. IEEE Journal of Oceanic Engineering, 38, 655-665.

Porter, M. B. ve Bucker, H.P., 1987. Gaussian Beam Tracing for Computing Ocean Acoustic Fields. Journal of Acoustical Society of America, 82, 1348-1359.

Qarabaqi, P ve Stojanovic, M., 2013a. Statistical Characterization and Computationally Efficient Modeling of a Class of Underwater Acoustic Communication Channels. IEEE Journal Oceanic Engineering, 38, 701-717.

Qarabaqi, P. ve Stojanovic, M. 2013b., Acoustic Channel Simulator, http://millitsa.coe.neu.edu/?q=projects.

Qarabaqi, P. ve Stojanovic, M., 2009. Statistical modeling of a shallow water acoustic communication channel, Proceedings of $3 \mathrm{rd}$ underwater acoustic measurements conference, June 2009, Nafplion, Greece, s. 1341-1350.

Qarabaqi, P. ve Stojanovic, M., 2011. Modeling the large scale transmission loss in underwater acoustic channels, Proceedings of 49th Annual Allerton Conference on Communication, Control and Computing, September 2011, Illinois, USA, s. 445-452.

Radosevic, A., Proakis, J. ve Stojanovic, M., 2009. Statistical characterization and capacity of shallow water acoustic channels, Proceedings of IEEE Oceans Europe Conference, May 2009, Bremen, Germany, s. 1-8.

Socheleau, F., Passerieux, J. ve Laot, C., 2009. Characterisation of timevarying underwater acoustic communication channel with application to channel capacity, Proceedings of 3rd underwater acoustic measurements conference, June 2009, Nafplion, Greece, s. 1-8.

Stefanov, A. ve Stojanovic, M., 2011. Design and performance analysis of underwater acoustic networks. IEEE Journal on Selected Areas in Communications, 29, 2012-2021.

Tamazin, M., Noureldin, A., Korenberg, M.J. ve Kamel, A.M., 2016. A New High-Resolution 
GPS Multipath Mitigation Technique Using Fast Orthogonal Search. Journal of Navigation, 69, 794-814.

Tomasi, B., Casari, P., Badia, L. ve Zorzi, M., 2010. A study of incremental redundancy hybrid ARQ over Markov channel models derived from experimental data, Proceedings of the 5th ACM International Workshop on UnderWater Networks, October 2010, Massachusetts, USA, s. 1-8.

United Nations, 2017. The First Global Integrated Marine Assessment: World Ocean Assessment: Cambridge University Press, 973 p.

Wang, W. B. ve Yang, T.C., 2006. High-frequency channel characterization for -ary frequencyshift-keying underwater acoustic communications. Journal of Acoustical Society of America, 120, 2615-2626.

Wang, Y., 2015. Linear least squares localization in sensor network. EURASIP Journal on Wireless Communications and Networking, 51, 1-7.
Yang, J. ve Chen, Y., 2009. Indoor Localization Using Improved RSS-Based Lateration Methods, Proceedings of the 28th IEEE conference on Global telecommunications, November 2009, Hawaii, USA, s. 4506-4511.

Zekavat, R. ve Buehrer, R.M., 2011. Handbook of Position Location Theory Practice and Advances: Wiley-IEEE Press, 1222 p.

Zhang, J., Cross, J. ve Zheng, Y.R., 2010. Statistical channel modeling of wireless shallow water acoustic communications from experiment data, Proceedings Military Communications Conference, November 2010, California, USA, s. 2412-2416.

Zhao, X., Pompili, D. ve Alves, J. 2017., Underwater Acoustic Carrier Aggregation: Achievable Rate and Energy-Efficiency Evaluation. IEEE Journal of Oceanic Engineering, 42, 1035-1048. 